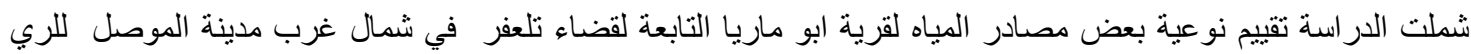

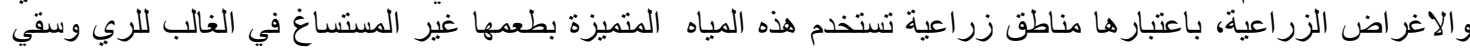

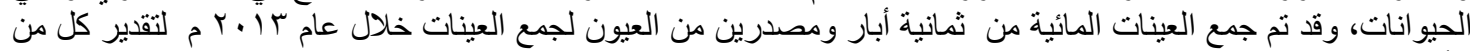

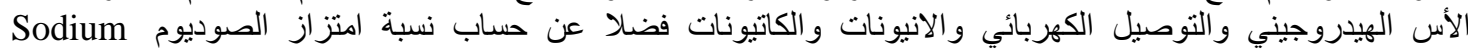

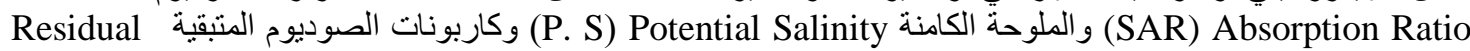
(RSC)Sodium Carbonate لتقييم نوعية المياه للأغر اض المختلفة، فضلا عن تقييم المياه لأغراض اض الري الري وسقي المو اشي بالاستناد إلى التصانيف المعتمدة

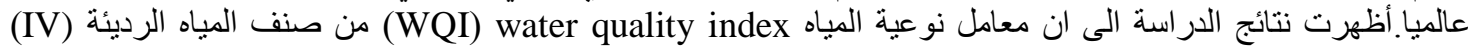

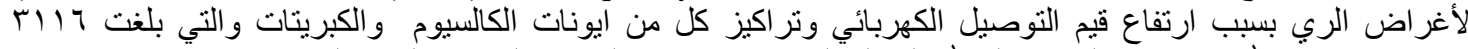

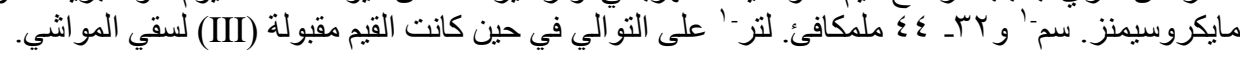

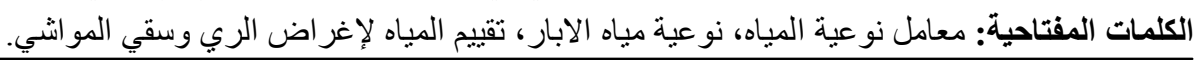

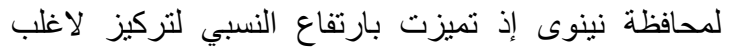

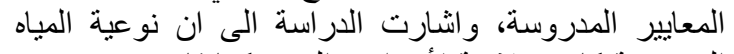

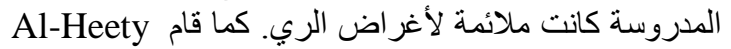
بإيجاد معامل نوعية المياه (et al., 2011)

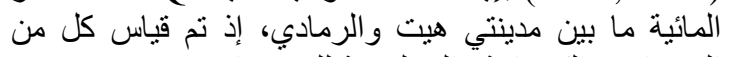

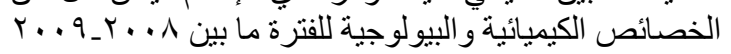

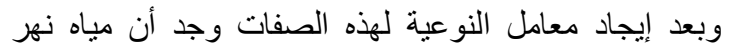

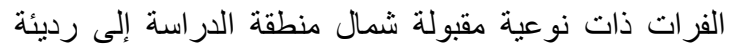

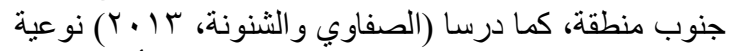

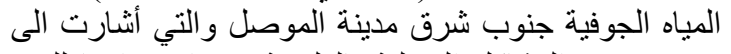

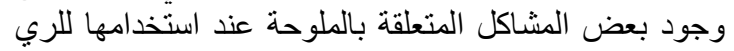
حسب التصانيف القياسية العالمية المعتمدة وكانت نتائج معائة معامل

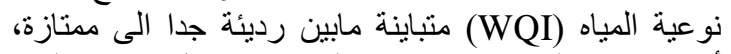

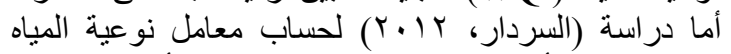

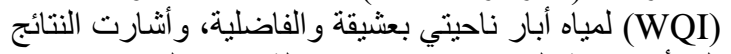

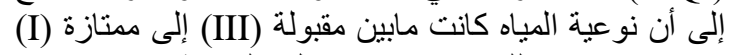

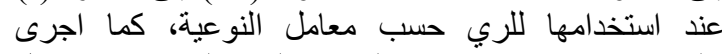

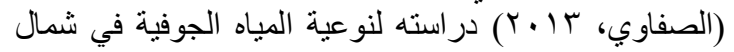

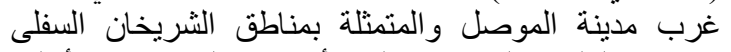

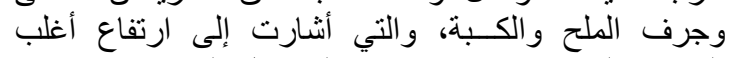

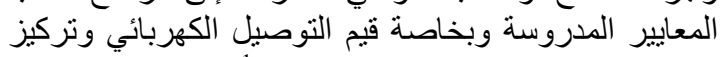

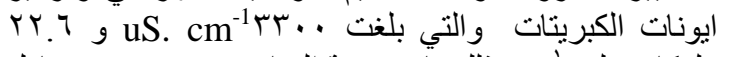

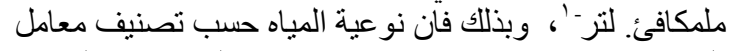

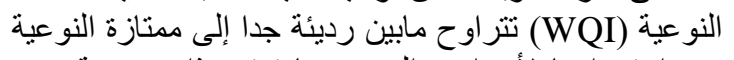

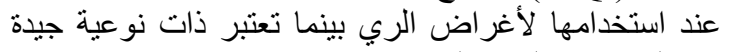
جدا إلى ممتازة لسقي الحيو انات.

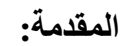

أصبحت مشكلة التلوث البيئي من أكثر القضايا حساسية في

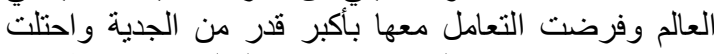

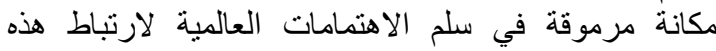

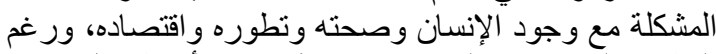

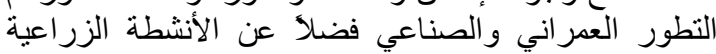

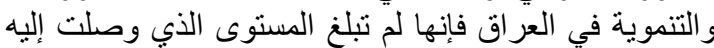

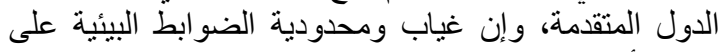

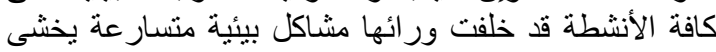

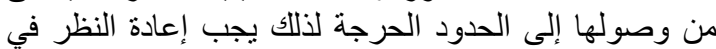

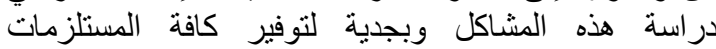

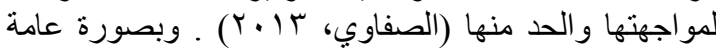
تحتوي المياه الجوفية على تراكيز عالية نسبيا من الاملاح

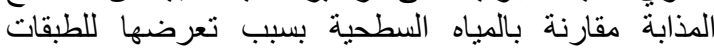

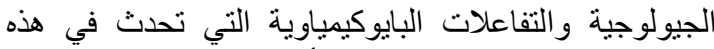

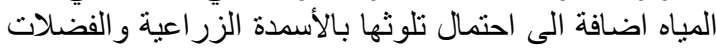

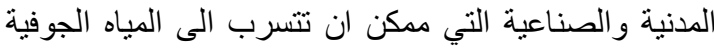
عبر الطبقات النفاذة (Al-Saffawi et al, 2018).

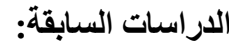
اجريت الكثير من الدراسات البهات على مصادر المياه في العراق

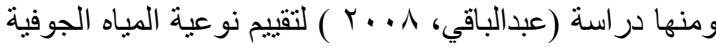

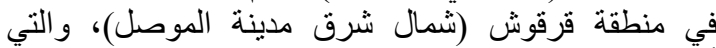

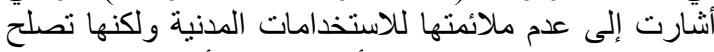

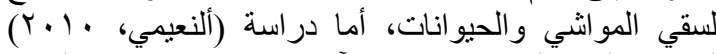

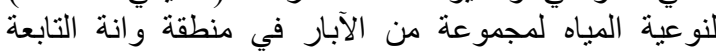




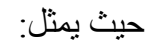
:WQI F Fi $=F_{i}\left(p_{i}\right)$

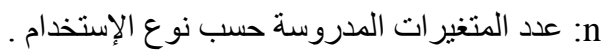

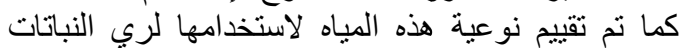

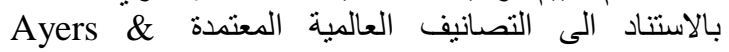
(Branson, 1977; Richard, 1969) النتائج والمناقشة:

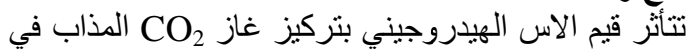
الماء وكبريتيد الهيروجين وايونات الكلوريد و البيكاربونات فئر . . الخ (Krupa and Parikh, 2018). وتشير النتائج المبينة في الجدول (r) الى التذبذب النب النسبي

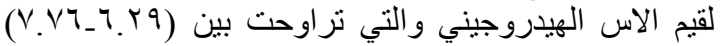
وتعود القيم القاعدية قليلا الى وجود التين ايونات البيكاربونات

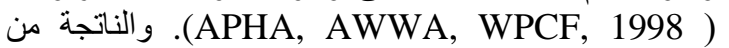
التفاعلات البايوكيميائية بين الغاز ات وكبريتات الكالسيوم

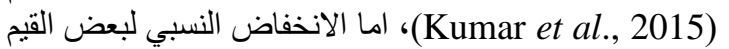
قد يعود الى ارتفاع تركيز الاملاح وسيادة الطور الكلوريدي لإني

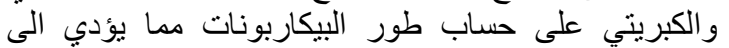

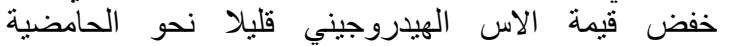

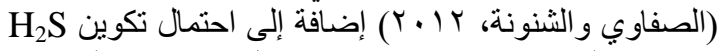

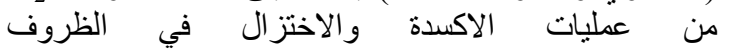

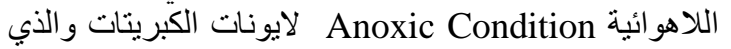

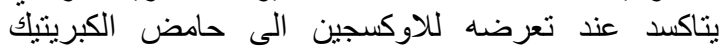

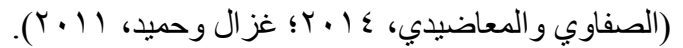

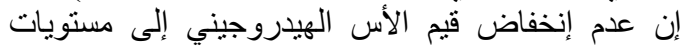
منخفضة جداً رغم هذه العمليات يعود إلى قابلية تأثير المياه

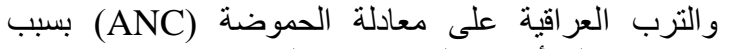

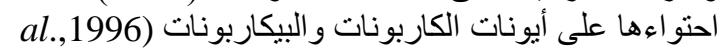
(Al-Saffawi and Al-Molaa, 2018؛ كذلك يلاحظ ارتفاع معدل قيم التوصيل الكهربائي والمبين

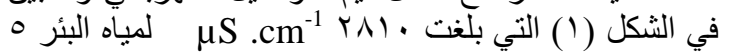

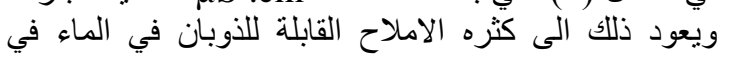

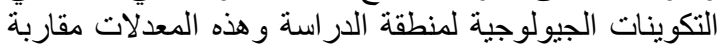

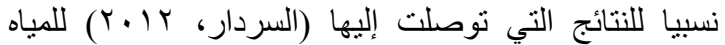

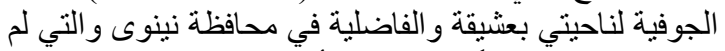

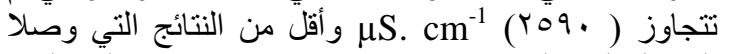

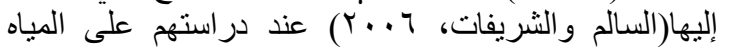

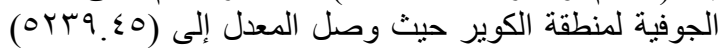
. $\mu \mathrm{S} . \mathrm{cm}^{-1}$

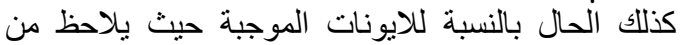

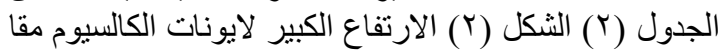

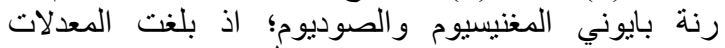

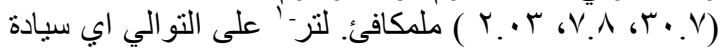

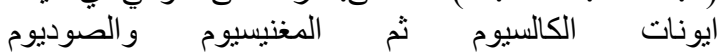

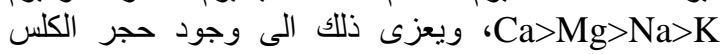
و الدولومايت وكبريتات الكالسيوم. اما الايونات السالبة فيلاحظ الاتيوم التفاع تركيز ايونات الكبريتات في جميع الابار المدروسة نتيجة للطبيعة

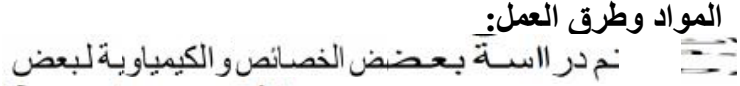

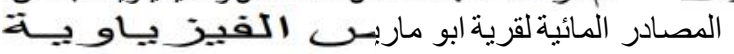
نينوى شمال غرب مدينة الموصل وائلة الموضح فئ المخطط (1)

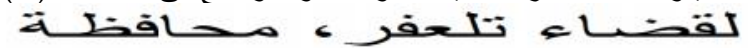

الانهار و المسطحات المائية ، حيث تعد مياه الابار المدروسة

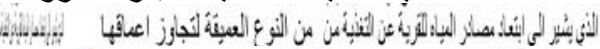

$$
\begin{aligned}
& \text { • } \\
& \text { المباه المدروسة. }
\end{aligned}
$$

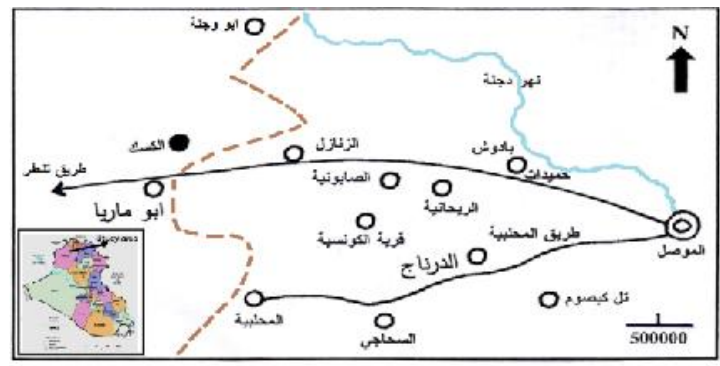

\begin{tabular}{|c|c|c|c|c|}
\hline \multicolumn{5}{|c|}{ 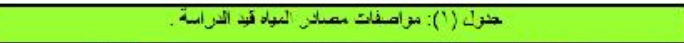 } \\
\hline الونَفْكاك & نوع انمهضر & 20 & السم حساهب أنبرّك & $\Xi$ \\
\hline 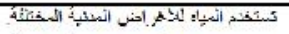 & بُّر. & 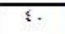 & مني دحسن حسن & 7 \\
\hline 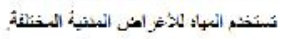 & بنز & s. & ظليث اير أشيه & $r$ \\
\hline 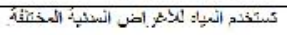 & $\dot{4}$ & $\varepsilon 0$ & صنيح حسر حسز & $T$ \\
\hline 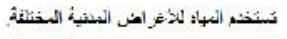 & بنز & 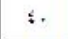 & ل ذافلـل العبايسي & $\div$ \\
\hline 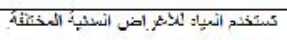 & بؤر & r & لي فل سحتد لـلن & c \\
\hline 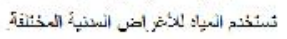 & بن. & r & 3. & 7 \\
\hline 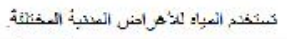 & ن & $\leqslant 7$ & نيلر صناح & v \\
\hline 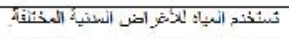 & بثن & \&0 & 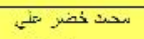 & $\sqrt{n}$ \\
\hline 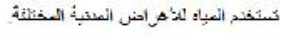 & 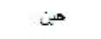 & $\cdots$ & معن لنبصة & 9 \\
\hline يناه في الثقربة & & -- & انتين أكبيد3 & \\
\hline
\end{tabular}

مخطط (1) ) موقع الدر اسة للمصادر المائية لقرية ابو ماريا.

تتميز منطقة الدراسة بوجود تكوين الفتحة (الفارس

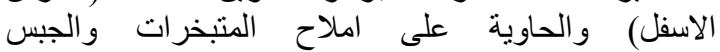

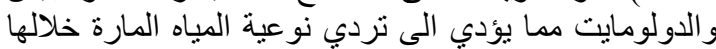

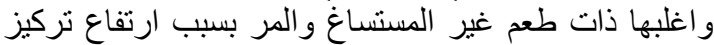

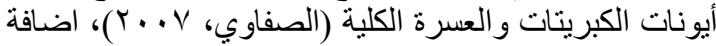
الى الاضرار الاقتصادية الناتجة من استخدام مثل هذه المئل المياه

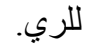

تم جمع العينات المائية ابتداء من شهر شباط ولغاية تشرين

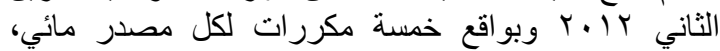
و اتبعت الطرق القياسية في جمع العينات وتحلئه

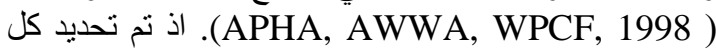
من الأس الهدروجيني والتوصيلية الكهربائية والايونات

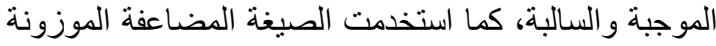
و المعروفة بصيغة (Multiplicative Weighted Mean) الوسط الهندسي (Geometric Mean) في ايجاد معامل

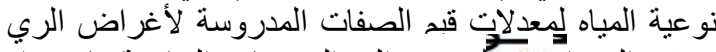

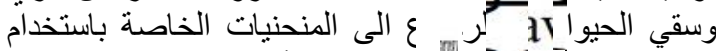

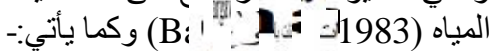

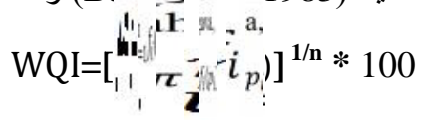


ارتفاع تركيز جها الملوحة Potential Salinity والتئي

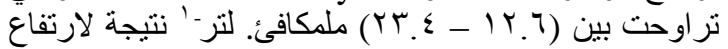

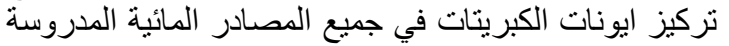

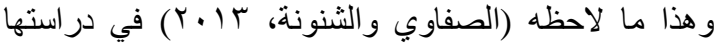

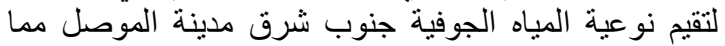

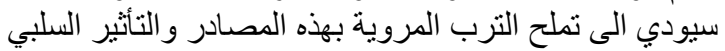

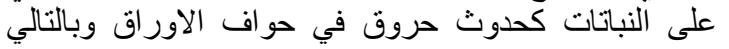

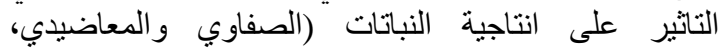

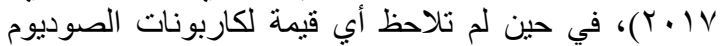

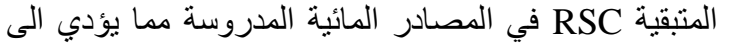

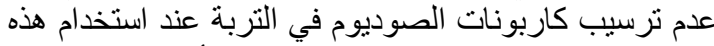

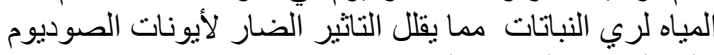

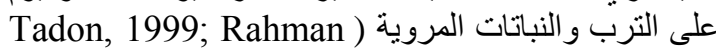
.(\& Chughtai, 2014
الجيولوجية للمنطقة والتي تحتوي على كبريتات الكالسيوم

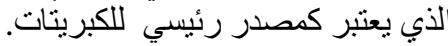

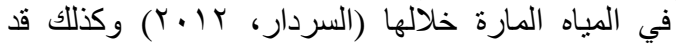

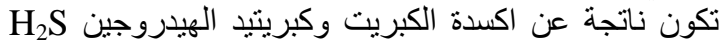

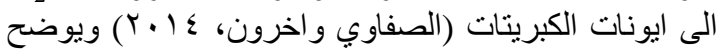

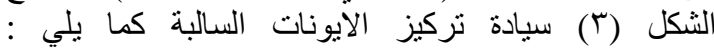
و هذه السيادة قد اكدتها الدراسات ألتي

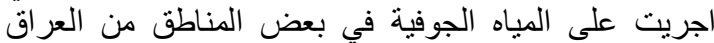

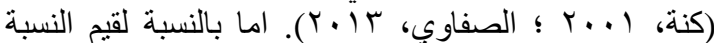

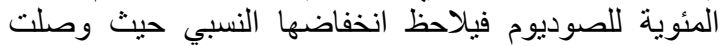

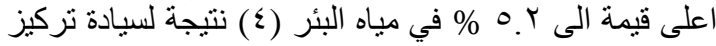

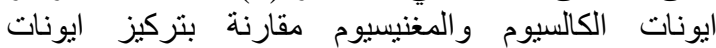

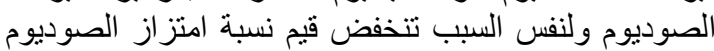

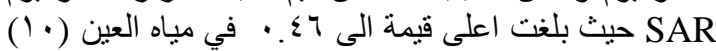

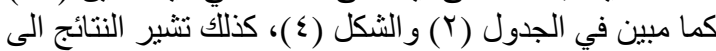

\begin{tabular}{|c|c|c|c|c|c|c|c|c|c|c|}
\hline \multicolumn{11}{|c|}{ 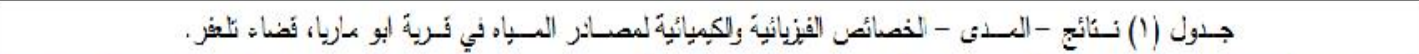 } \\
\hline 1. & q & $\wedge$ & r & 7 & • & $\varepsilon$ & r & r & 1 & \\
\hline $\max \min$ & $\max \min$ & $\max \min$ & $\max \min$ & $\max \min$ & $\max \min$ & $\max \min$ & $\max \min$ & $\max \min$ & $\max \min$ & \\
\hline $7.0 \quad 6.32$ & $7.76 \quad 6.34$ & $7.15 \quad 6.52$ & $7.58 \quad 6.29$ & $7.37 \quad 6.37$ & 7.176 .78 & 7.166 .47 & $7.43 \quad 6.37$ & $7.28 \quad 5.41$ & 7.296 .40 & $\mathrm{pH}$ \\
\hline 31162277 & 27712021 & 28092156 & 30012252 & 31292368 & 30012651 & 29752223 & 27462044 & 28092115 & 28092125 & $\mathrm{Ec}_{2: m S \mathrm{~m}}$ \\
\hline rr Yq & Yq Y Y & Yq YY & Tr T. & ry rt & ry YA & 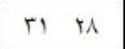 & rr r. & rr YV & rr $\quad+1$ & $\mathrm{Ca}$ meq! \\
\hline A.1 $\quad 5.7$ & I.Y T.. & ר. & 0.7 r.\& & 1. T. & A., $7 . \varepsilon$ & A.t c. c. & T.1 Y.Y & C.Y T.. & T.Y T.Y & $\mathrm{Mg}$ meq $l$ \\
\hline r.. $\quad 1.7$ & 1.. $\quad .1$ & 1. $\quad .9$ & I.V $\quad$ 1. & r.I $\quad$ I.r & $\begin{array}{lll}1 . y & 1 . V\end{array}$ & $\psi . . \quad 1 . \psi$ & 1.r $\quad 1.1$ & $1.1 \quad \ldots$ & l.r $\quad$... & $\mathrm{Na}$ meq! \\
\hline$\therefore$ & $\ldots \circ \quad \ldots \varepsilon$ & 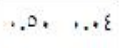 & $\ldots 0 \quad \ldots 0$ & .r. . ..1. & $. .1 r \quad . .1 r$ & $\ldots 0 \quad \ldots$ & $\ldots$ & $\ldots \varepsilon \quad \ldots r$ & . . . . . & $\mathrm{K}$ meq $/$ \\
\hline re rI & ri ri & r. rq & rq $r$. & rA ri & $\begin{array}{ll}r \Lambda & \text { r }\end{array}$ & ry YC & TA KT & 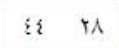 & Tr TI & $\mathrm{SO}_{4}$ meq $\mathrm{l}$ \\
\hline T.r T.V & T.1 Y.r & r.h r. r. & T.T r.Y & $r .1 \quad \psi . V$ & T.T r.I & T.q $\quad \mathrm{r.}$ & r.\& & r.Y T.I & r.. ү.. & $\mathrm{HCO}_{1}$ meq $/$ \\
\hline$r .0 \quad 1.5$ & $1.8 \quad .0$ & $1 . Y \quad . .1$ & r.1 $\quad 1.7$ & r.r $\quad$ r. & r.T $\quad 1.0$ & $4.7 \quad 1.1$ & ع. 1.7 & $1.1 \quad .1$ & $1.5 \quad .11$ & $\mathrm{Cl}$ meql \\
\hline 17.911 .1 & $17 . \wedge$ וr. & $17.8 \quad 17.5$ & +1.21 .1 & r.c M. M & $17.8 \quad 18.7$ & $16.7 \quad 10 . \varepsilon$ & ז... & rT. $10 . \mathrm{r}$ & r. t IV.. & P.S meq! \\
\hline c., $\quad$ l.. & r.r $\quad . .1$ & $r .1 \quad \cdots$ & E.r $\quad 1.9$ & $\varepsilon . . \quad$ i.V & $\quad \ldots \quad 1 .$. & $0 . r \quad$... & r.T $\quad .1$ & $\varepsilon .1 \quad 1$. & r. $\quad$ i.. & $\mathrm{Na} \%$ \\
\hline..$\{7 \quad . Y$ & $\ldots 11 \quad \ldots 9$ & r... & .s. tro & 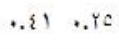 & I.t. A. T. &. Tr . . V V & $.{ }^{r}, \quad .10$ &. .YA $\quad . . Y$ & $. . \quad . .1 \varepsilon$ & SAR \\
\hline$\cdots$ & $\cdots$ & $\cdots$ & $\ldots$ & .. & $\cdots$ & $\ldots$ & $\cdots$ & $\cdots$ & $\ldots$ & RSC \\
\hline
\end{tabular}

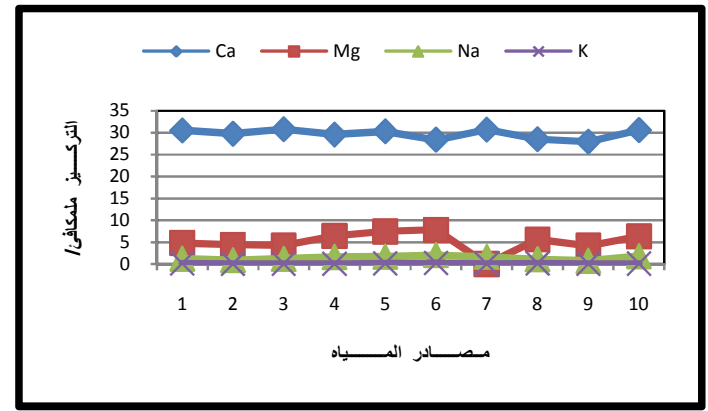

الثكل (ץ): معدل تر اكيز الايونات الموجبة للمصادر المائــــية

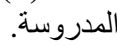

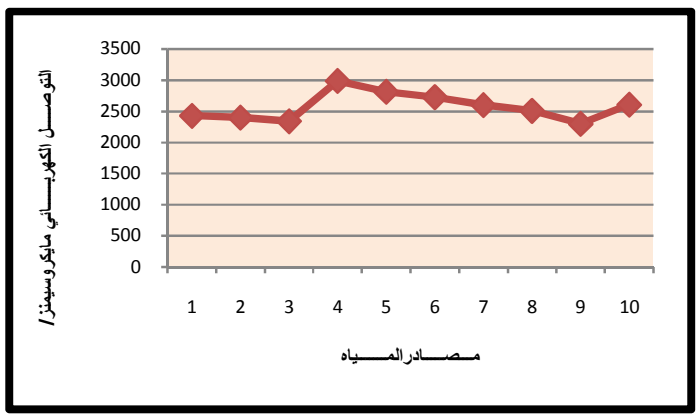

الثكل (1): معدل قيم التوصيل الكهربائي للمصادر المائــية المدروسة. 


\section{تقيم نوعية مياه الآبـــار:} تعد جميع المصادر المائية المدروسة الآبار المدروسة وحسب معامل نوعية المياه WQI مقبولة النوعية (III) عند المبانة

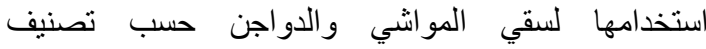

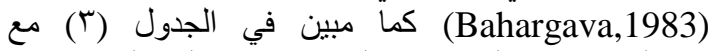

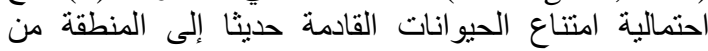

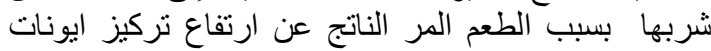

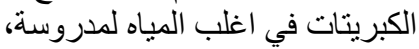

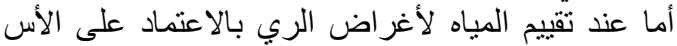
الهيدروجيني والتوصيل الكهربائي وتركيز ايونات الكيات الكلوريد

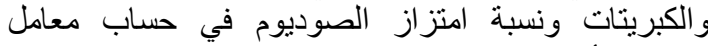

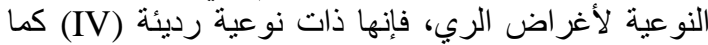

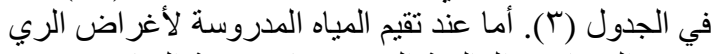

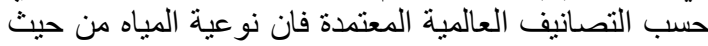

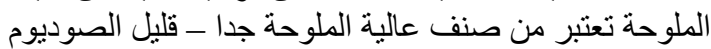
US. حسب تصنيف مختبر الملوحة الأمريكي $C_{4} S_{1}$ .Salinity Lab

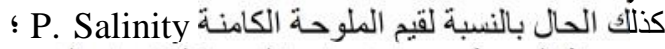

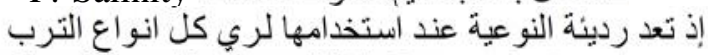

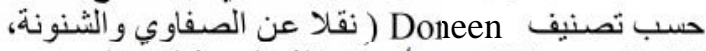

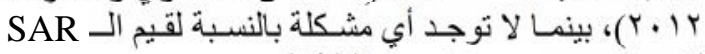

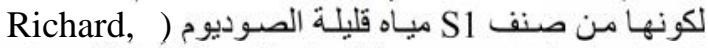

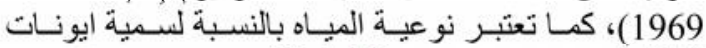

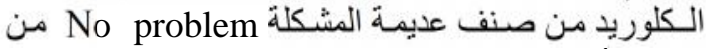
حيث التأثير على نفاذية التربة و السمية على النباتات المروية حسب تصنيف (1977، Ayers \& Branson).

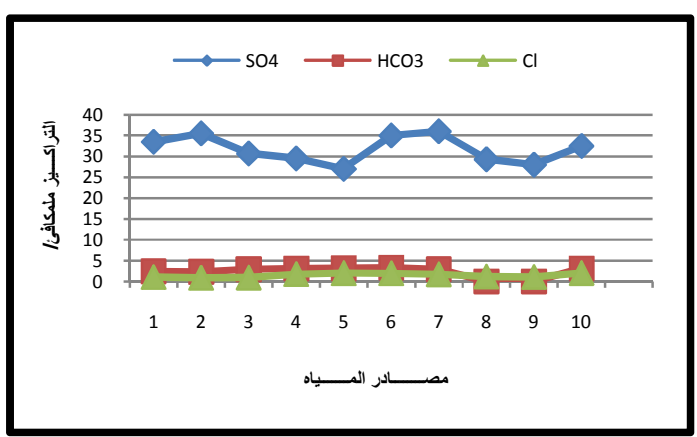

الشكل (r): معدل تراكيز الايونات السالبة للمصادر المائــــية المدروسة.

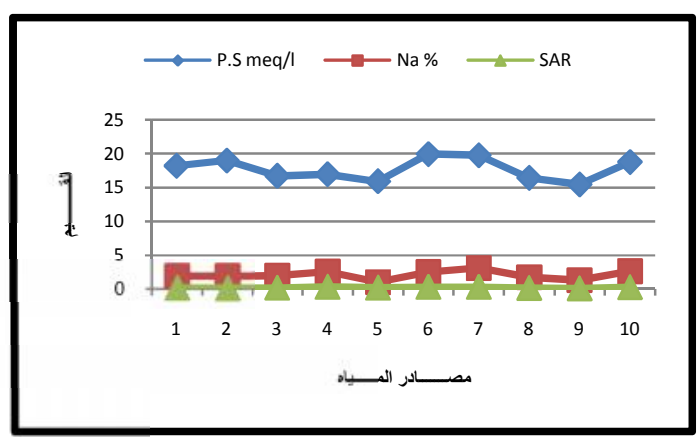

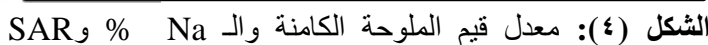
للمصادر المائية المدروسة.

\begin{tabular}{|c|c|c|c|c|c|c|c|}
\hline & & سقي الم & لمدرو & صنانر & ن تص & & \\
\hline \multirow{3}{*}{$R S C$} & \multirow{3}{*}{ P. Sal. } & \multirow{3}{*}{$E C-S A R$} & \multicolumn{4}{|c|}{$W Q I$} & \multirow{3}{*}{$\mathrm{NO}$} \\
\hline & & & \multicolumn{2}{|c|}{ سفي المو انثي } & \multicolumn{2}{|c|}{ للـــري } & \\
\hline & & & الَّفِمة & الثنو عية" & ألهِيمة & اللنو عية & \\
\hline ممثازة & رديئة & $C_{4} S_{1}$ & 43.3 & مقُبولة & 27.7 & رديئة (IV) & 1 \\
\hline ممثازة & رديئة & $\mathrm{C}_{4} \mathrm{~S}_{1}$ & 42.0 & مقبولة & 26.5 & رديئة (IV) & T \\
\hline ممثازة & رديئة & $\mathrm{C}_{4} \mathrm{~S}_{1}$ & 41.0 & مقبولة & 28.7 & رديئة (IV) & $r$ \\
\hline ممثتزة & رديئة & $C_{4} S_{1}$ & 41.2 & مقبولة & 26.3 & رديئة (IV) & $\varepsilon$ \\
\hline ممثازة & رديئة & $\mathrm{C}_{4} \mathrm{~S}_{1}$ & 41.9 & مقبولة & 23.3 & رديئة (IV) & 0 \\
\hline ممثازة & رديئة & $\mathrm{C}_{4} \mathrm{~S}_{1}$ & 42.1 & مقببولة & 23.5 & رديئة (IV) & 7 \\
\hline ممثتزة & رديئة & $\mathrm{C}_{4} \mathrm{~S}_{1}$ & 42.0 & مقُبولة & 24.6 & رديئة (IV) & V \\
\hline ممثازة & رديئة & $\mathrm{C}_{4} \mathrm{~S}_{1}$ & 40.3 & مقبو لَة & 27.2 & رديئة (IV) & $\wedge$ \\
\hline ممثازة & رديئة & $\mathrm{C}_{4} \mathrm{~S}_{1}$ & 40.0 & مقُبو لهُ & 28.5 & رديئة (IV) & 9 \\
\hline ممتَزة & رديئة & $C_{4} S_{1}$ & 41.9 & مقفبولة & 26.5 & رديئة (IV) رئة & 1. \\
\hline
\end{tabular}

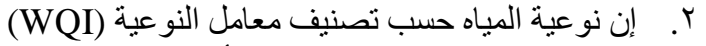

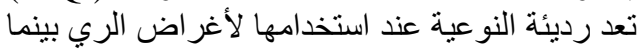

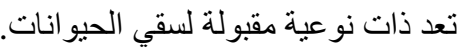

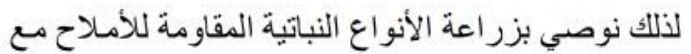

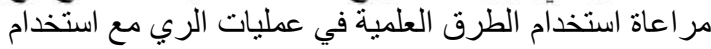
الطرق الحديثة في الري كطريقة الري بالتنقيط.
الاستتتاجات والتوصيات:

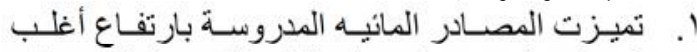

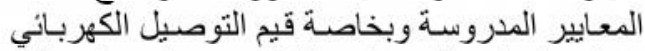

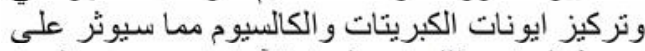
نوعية المياه وبذللك تعد المبياه الآبـار من صنئ صنف المبات المبياه

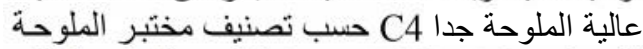

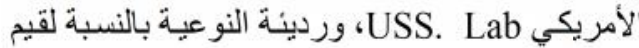
الملوحة الكامنة. 


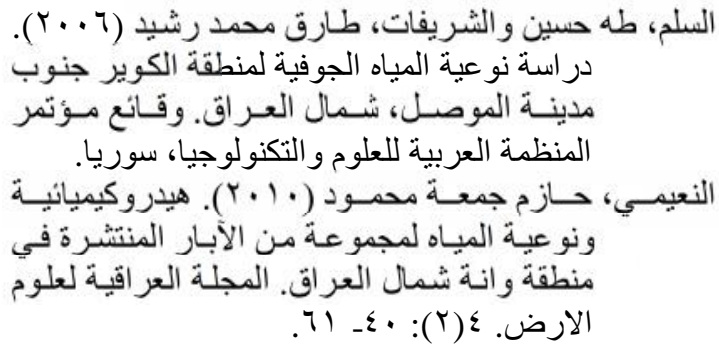

Al-Heety, E.A.M., Turki, A.M. and AlOthman, E.M. (2011). Assessment of the water quality index of Euphrates river between Heet and Ramadi Cities. Inter. J. Basic Appl. Sci 11(6): 38-47.

Al-Saffawi, A.A.Y.T., Al sinjari, W.E. and AL-Taee, Y.A.J. (2018 b). Assessment of groundwater quality using (WQI) in Gleewkhan village northeastern of Iraq. Int. J. Enhanced Res. In Sci.,Tech. \& Engin. 7(5): 1 - 7.

Al-Saffawi, A.Y.T., Al-Molaa, Y.T.M. (2018). Quality characterization of groundwater by using water quality index in Al- Kasik district Northeastern of Mosul City. Iraq. Int. J. of Enhanced Res. in Sci., Techn. \& Engin. 7(1):76-81.

Al-Saffawi, A.A.Y.T. and Al-Sardar, N.M.S. (2018). The possibility of some physical and biological methods to improve the groundwater quality. Educat. \& Sci. J. for Pure Sci, 27(2): 47-60.

APHA. AWWA. WPCF. (1998). "Standard methods for examination of water and wastewater". $20^{\text {th }}$ ed., Washington DC. USA.

Ayers, R.S. and Branson, R.L. (1977). Water quality guide line for interpretation of water quality fractures. Univ. California Comm.Con. California Agric., 31:250.

Bahargava, D.S. (1983). Use of Water quality index for river classification and zoning of Ganganga River. Env. Pollut. Ser. B. An Inter. J. England 6:51-67.

Dokmen, F. and Yuksel, A.N. (2007). An investigation in to the quality of irrigation water from underground water in the vicinity of the coast of

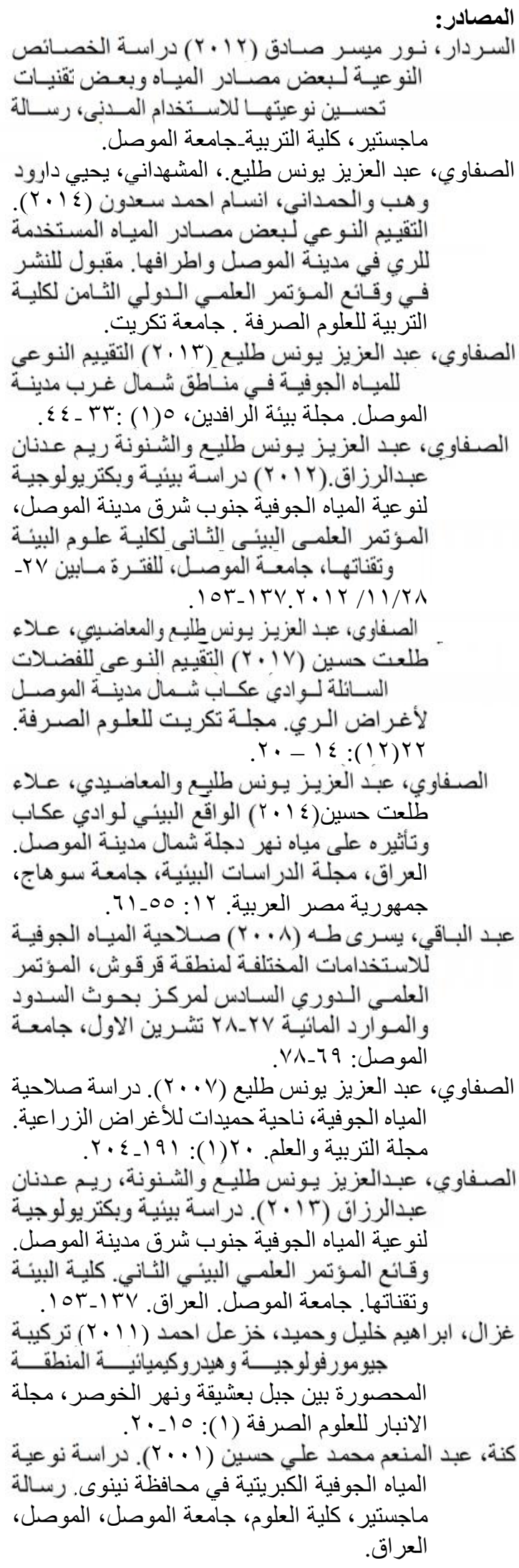


quality data using regression model Afri. J. Envir. Sci \& Tech., 8(1): 86: 90.

Richerd, A. (1969). "Diagnosis and Improvement of Saline and Alkali Soil", USDA Hand Book No. 60, 160.

Tadon, H. (1999). "Methods of analysis of Soil ,Plant, Water and Fertilisers, Binny printers, New Delhi, India.

Weng, J.H., Huang, L.F., Liu, X. and Sato, K., (1996). Acid neutralizing capacity of some typical forest surface soil in China, Proc. CRIEPI Int'l seminar on transport and effects of acidic substances, p:154-158, Nov. 28-29, Tokyo, Japan.
Yalva, Turky. Word J. Agric. Sci. 3 (1): 105-110.

Krupa, U. and Parikh, P. (2018). Water quality index of harni pond, district Vadodara, Gujarat Int. J. Allied. Pract. Res. And Rev. (IJAPRR). 7(1):6-1.

Kumar, S.K., Logeshkumaran, A., Magesh, N.S., Prince, S. Godson, P.S. and CHandrasekar, N. (2015). Hydrogeochemistry and application of water quality index (WQI) for ground water quality assessment, Anna Nagar part of Chennai city, Tamil Nadu, India. Appl. Wat. Sci., 5:335-343.

Rahman, A. and Chughtai (2014). Reginol interpretation of river Indus water

\title{
Water Quality Assessment for irrigation and livestock drinking in Abu Maria village/ district of Tall-Afar-Iraq
}

Reem Adnan Abdul-Razaq Al-Shanona, Noor Myasar Sadeq, Abdul-Aziz Y.T. Al-Saffawi

\begin{abstract}
The objective of this study was to evaluate the quality of some water resources in the location of Abu Maria village in the district of Tall Afar in the north - eastern of Mosul city for irrigation and agricultural uses, which is considered as an agricultural land which uses these water resources for livestock drinking and agricultural irrigation, water samples were collected from eight wells and two spring during 2013 to determine some parameters such as $\mathrm{pH}, \mathrm{EC}_{25}$, Anions, Cations, SAR, RSC and P. Salinity, the geometric mean formula was applied to determination of water quality index (WQI) for evaluation, also the water resources was evaluated for agricultural uses according to universal standard classification. The results of water quality index (WQI) revealed that water resources under study have bad quality ( category IV) due to high value of $\mathrm{Ec}_{25}, \mathrm{Ca}$ and $\mathrm{SO}_{4}{ }^{=}$which amount to $3116 \mu \mathrm{S} / \mathrm{cm}$, and 32-44 meq. $/ 1$ respectively, but it have suitable quality for livestock drinking (category III).
\end{abstract}

Key wards: WQI, Ground water quality, Water assessment for agricultural uses. 